\title{
Carrier multiplication in bulk indium nitride
}

\author{
S. A. Jensen, ${ }^{1,2}$ J. Versluis, ${ }^{1}$ E. Cánovas, ${ }^{2}$ J. J. H. Pijpers, ${ }^{1, a)}$ I. R. Sellers, ${ }^{3}$ and M. Bonn ${ }^{2}$ \\ ${ }^{1}$ FOM Institute for Atomic and Molecular Physics, Science Park 104, 1098 XG Amsterdam, The Netherlands \\ ${ }^{2}$ Max Planck Institute for Polymer Research, Ackermannweg 10, 55128 Mainz, Germany \\ ${ }^{3}$ Homer L. Dodge Department of Physics and Astronomy, The University of Oklahoma, \\ 440 W. Brooks St. Norman, Oklahoma 73019, USA
}

(Received 6 August 2012; accepted 25 October 2012; published online 30 November 2012)

Carrier multiplication $(\mathrm{CM})$ is the process of generating multiple electron-hole pairs from one absorbed photon. Narrow-gap InN is a material that has been proposed for achieving efficient CM. We quantify the CM efficiency in bulk InN using terahertz time-domain spectroscopy. While the $\mathrm{CM}$ onset occurs at relatively low photon energies in $\mathrm{InN}(1.7 \pm 0.2 \mathrm{eV})$, corresponding to $2.7 \pm 0.3$ times its bandgap, the excitation efficiency above the onset increases linearly with a slope of only $\sim 13 \% / E_{g}$. Based on these numbers, the efficiency increase of an $\mathrm{InN}$ based photovoltaic device owing to $\mathrm{CM}$ is limited to maximum 1\% point. (c) 2012 American Institute of Physics. [http://dx.doi.org/10.1063/1.4766738]

Carrier multiplication $(\mathrm{CM})$ is the process by which the excess energy of a photoexcited electron (or hole) in a semiconductor is used to promote additional electrons across the bandgap. CM has been explained in terms of impact ionization, ${ }^{1,2}$ and different studies have consistently shown that $\mathrm{CM}$ is present in many bulk semiconductor materials ${ }^{1,3,4}$ and nanostructures. ${ }^{5} \mathrm{CM}$ can, in principle, be exploited for boosting solar cell efficiencies beyond the Shockley-Queisser limit $^{2,6}$ under, otherwise, identical conditions, as it increases the potential solar cell photocurrent while reducing thermalization losses. Theoretical studies have shown that the maximum efficiency achievable by CM is $44.4 \%$ for an ideal absorber material with an optical bandgap of $0.7 \mathrm{eV}^{2,7} \mathrm{InN}$ is a promising candidate for $\mathrm{CM}$ based photovoltaics as it was recently discovered to have a bandgap close to 0.7 $\mathrm{eV}^{8,9}$ In addition, InN fulfills two other requirements for high CM performance: ${ }^{2}$ (i) a wide phononic bandgap, ${ }^{10}$ expected to reduce losses caused by phonon emission and (ii) an asymmetric valence and conduction band structure ${ }^{11}$ that results in a large electron/hole effective mass-mismatch. This mismatch leads to an excess photon energy that is preferentially transferred to the electron, rather than the hole. ${ }^{12}$ Such characteristics offer the potential for $\mathrm{InN}$ to reach high photo-conversion efficiencies via CM.

Here, the CM efficiency in bulk $\mathrm{InN}$ is quantified. The investigated sample is a monocrystalline $0.5 \mu \mathrm{m}$ thick $\mathrm{InN}$ layer grown by molecular beam epitaxy on the lattice matched wide bandgap materials; $\mathrm{GaN}(0.2 \mu \mathrm{m})$ and Be:GaN $(0.1 \mu \mathrm{m})$. Photoluminescence and optical absorption measurements revealed an optical bandgap for the $\mathrm{InN}$ film of less than $0.7 \mathrm{eV}$, indicating an intrinsic doping concentration on the order of $10^{18} \mathrm{~cm}^{-3}$, which is comparable to the lowest reported values for InN. ${ }^{13,14}$ The surface of the sample was pure, unoxidized $\mathrm{InN}$ as verified by energy dispersive $\mathrm{x}$-ray spectroscopy (EDX).

Carrier multiplication efficiencies vs excitation wavelength were quantified using $\mathrm{THz}$ time domain spectroscopy

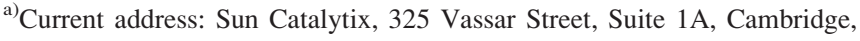
Massachusetts 02139, USA.
}

(THz-TDS). ${ }^{15}$ In this pump-probe technique, charge carriers are promoted to the conduction band $(\mathrm{CB})$ by an optical pulse of femtosecond duration and probed by a single cycle THz pulse (comprising of frequencies in the $0-2 \mathrm{THz}$ range). As $\mathrm{THz}$ radiation is sensitive primarily to mobile charge carriers in bulk semiconductors, ${ }^{15}$ the photoinduced absorption of the $\mathrm{THz}$ field reveals the photoconductivity of the sample on ultrafast timescales in a contact free manner. In this way conductivities can be inferred locally without moving charges over large distances, as is the case in device photocurrent measurements. Measuring photoconductivities on ps timescales after excitation also means avoiding complications caused by radiative recombination and recombination at surfaces and interfaces. ${ }^{4}$ For the determination of $\mathrm{CM}$ efficiencies, the excitation photon flux needs to be determined very reliably. To this end, a homogeneous impinging flux of pump photons over the sample area was ensured using an optical diffuser, the fluence of which was accurately determined by a calibrated detector and five calibrated apertures of increasing size. The optical absorption of the sample was independently determined, and transmission losses of the pump beam were corrected for. Reflection losses were also considered using reported values for the $\mathrm{InN}$ complex dielectric function. ${ }^{16}$

The photoconductivity measurement described in Ref. 15 involves measuring the photoinduced change in transmitted $\mathrm{THz}$ field $\left(\Delta T_{\text {photo }}\right)$ and the total $\mathrm{THz}$ transmission through the unexcited sample $\left(T_{\text {unexc }}\right)$ for reference. The ratio $\Delta T_{\text {photo }} / T_{\text {unexc }}$ is proportional to the sample's photoconductivity, which is determined by the product of the carrier density $N$, the elementary charge $e$, and the carrier mobility $\mu$. The photoconductivity is plotted in Figure 1 as a function of pump-probe delay for various excitation photon energies. As excitation occurs with an optical pump pulse duration of less than $100 \mathrm{fs}$, effectively instantaneous in our measurement, the gradual rise in $\mathrm{THz}$ absorption implies a time-dependent increase in the product $\mu \times N$ after the initial excitation.

An increase in $\mu$ can be explained by two separate mechanisms: First, as $\mu$ is inversely proportional to the carrier effective mass $m^{*}$, a gradual increase in $\mu$ can be understood by considering changes in $m^{*}$. Carriers are initially 


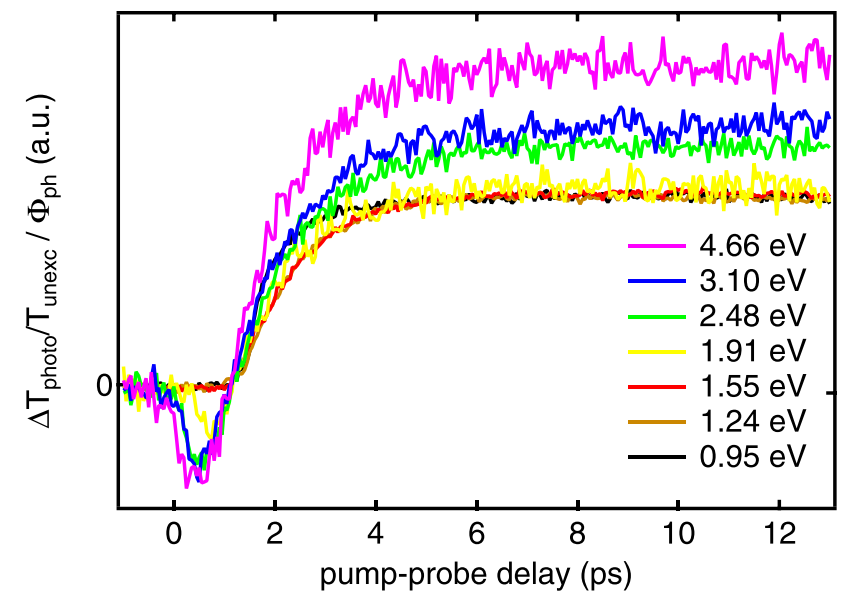

FIG. 1. Photoinduced THz absorption $\Delta T_{\text {photo }}$ divided by total transmitted $\mathrm{THz}$ intensity $T_{\text {unexc }}$ divided by photon fluence (photons $/ \mathrm{m}^{2}$ ) vs. pump delay at seven excitation wavelengths indicated by their corresponding photon energies. It is evident that for higher photon energies a larger signal per photon is observed, which is attributed to CM.

photoexcited well above the InN CB minimum. In these high energy states, $m^{*}$ is larger (than at the bottom of the CB) due to the non-parabolicity of the bands. ${ }^{14,17}$ Therefore, with increasing pump delay, a gradual cooling of the hot electrons results in a decrease in $\mathrm{m}^{*}$, consequently increasing $\mu$. Second, if a high carrier density is initially created within a thin layer of the sample, as can be the case when the pump energy is far above the absorption threshold, momentum randomizing elastic scattering events of excited electrons with holes and hot phonons can cause an initially lower $\mu$, which increases with time, as diffusion reduces the total charge density. ${ }^{18}$ The changing carrier density does not affect the measured photoconductivity directly (through $N$ ) as we are sampling the whole thickness of the InN layer. Additionally, the CM process itself is expected to generate secondary excited electrons after the initial excitation, causing an increase in $N$ over time after excitation. Presumably, the short-lived initial negative signal, observed just after excitation for pumping energies above $1.55 \mathrm{eV}$, can be related to stimulated $\mathrm{THz}$ emission from hot carriers in the InN layer. ${ }^{19}$ After $\sim 6$ ps the signal reaches a constant plateau for all pump energies indicating the absence of recombination events on this time scale. This is in agreement with the low defect concentration expected in our sample.

From Figure 1 it is clear that the magnitude of the photoconductivity per absorbed photon increases with increasing photon energy, which is indicative of CM. However, assessing the number of excited charge carriers per absorbed photon from the data shown in Figure 1 requires knowledge of the carrier mobility, $\mu . \mu$ can be determined from the complex conductivity of the photo-excited charge carriers as function of the probe (THz) frequency ${ }^{4}$ (see Figure 2). As is evident from Figure 2, the conductivity is well described using the Drude model for free carriers in a scattering medium under an electric field, oscillating at angular frequency, $\omega$ (black line in Figure 2)

$$
\sigma(\omega)=N \times e \times \mu(\omega)=N \times \frac{e^{2} \tau_{s}}{m^{*}} \times \frac{1}{1-i \omega \tau_{s}},
$$

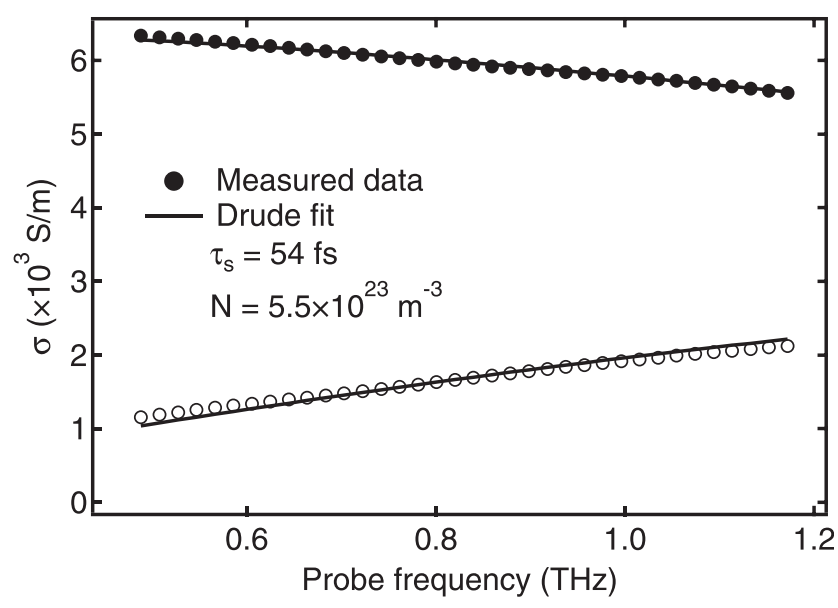

FIG. 2. Real (filled circles) and imaginary (open circles) parts of the photoconductivity vs. probe frequency measured $10 \mathrm{ps}$ after excitation by a 3.10 $\mathrm{eV}$ pump pulse of fluence $2.8 \times 10^{16} \mathrm{~m}^{-2}$ and fitted to Eq. (1). The density of excited electrons $(N)$ was extracted assuming an effective mass of $m^{*}=0.13 m_{0}$.

where $\tau_{s}$ is the mean time between momentum randomizing scattering events. By measuring photoconductivity versus probe frequency and fitting to Eq. (1) we found that $\tau_{s}$ has a value of $52 \pm 6 \mathrm{fs}$, independent of excitation energy and intensity (measurements were performed at $1.55 \mathrm{eV}, 3.10 \mathrm{eV}$, and $4.66 \mathrm{eV}$ at pump fluences $\Phi_{p h}$ ranging from $3 \times 10^{15}$ photons $/ \mathrm{m}^{2}$ to $1 \times 10^{17}$ photons $/ \mathrm{m}^{2}$ ). Thus $\mu$ is constant within the range of experimental conditions employed here, and the dependence of the magnitude of $\sigma(\omega)$ (i.e., $\Delta T_{\text {photo }}$ $T_{\text {unexc }}$ ) on excitation energy at long times (Figure 1) is caused only by variations in the carrier density. The fluence normalized conductivity $\Delta T_{\text {photo }} / T_{\text {unexc }} / \Phi_{p h}$ is therefore a direct measure of the efficiency of photo-excitation or quantum yield (QY). Assuming a 100\% QY at $1.55 \mathrm{eV}$, we find using Eq. (1) that the electron effective mass $m^{*}$ for our InN sample is $0.13 m_{0}$ (where $m_{0}$ denotes the free electron mass), which is consistent with previously reported values from $0.05 m_{0}$ to $0.24 m_{0} \cdot{ }^{14,20-27}$

The measurements shown in Figure 1 were performed at a range of excitation fluences (ranging from $2.9 \times 10^{15}$ photons $/ \mathrm{m}^{2}$ to $4.7 \times 10^{16}$ photons $/ \mathrm{m}^{2}$ ) for each excitation energy, and the signal magnitude was found to scale linearly with the excitation fluence, showing that carrier-carrier interactions are not significant. ${ }^{28}$ The slope of $\Delta T_{\text {photo }} / T_{\text {unexc }}$ vs. fluence is proportional to the quantum yield, plotted (black dots) versus pump photon energy in Figure 3.

Figure 3 shows that carrier multiplication is observed at photon energies from approximately $1.7 \pm 0.2 \mathrm{eV}$, which is between two and three times the value of the bandgap for InN. Above this value the QY rises linearly with photon energy with a slope of $21 \%$ of the pre-CM onset value per $\mathrm{eV}$, which is equal to a $13 \%$ increase per $E_{g}\left(E_{g}=0.64 \mathrm{eV}\right){ }^{9}$ Table I shows a comparison of the CM onset and slope efficiency measured for InN relative to other bulk semiconductor materials from previous works.

The experimental CM behavior for all materials in Table I is quite different from that of an ideal absorber (see blue line in Figure 3). Ideally, (i) CM should start at the lowest energetically allowed value, $2 E_{g}$, and (ii) the QY should increase by unity each time the excitation energy increases 


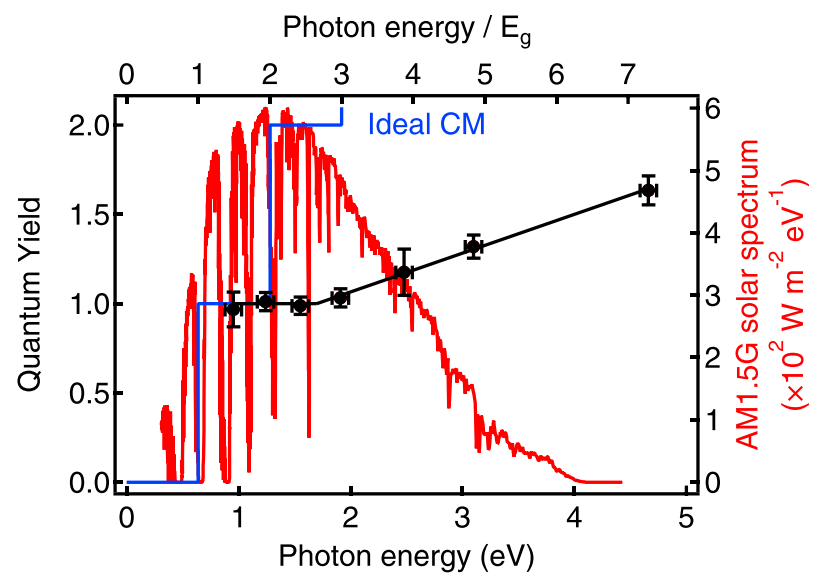

FIG. 3. Black dots show the measured QY vs. photon energy for InN (the black line is a guide to the eye). CM is observed at energies above $1.7 \pm 0.2$ $\mathrm{eV}$ equal to $\sim 2.7$ times InN $E_{g}$. In blue the QY of an ideal CM absorber ${ }^{7}$ with $E_{g}=0.64 \mathrm{eV}$ is shown, and in red, the AM1.5G solar spectrum.

by a unit of $E_{g}$. The causes for the deviation from ideal behavior are discussed in the following. Regarding (i), the delayed onset of $\mathrm{CM}$ (at $2.7 E_{g}$, rather than $2 E_{g}$ ) results from the excess photon energy being distributed over the initially excited electron and hole. For CM to occur, one or both charge carriers need to have surplus energy in excess of $E_{g}$ to facilitate a secondary interband transition over the bandgap. The total photon excess energy is distributed-to a first approximation-according to the electron and hole effective masses: the lighter particle receiving proportionally more energy than the heavy particle. ${ }^{12}$ Thus, in the case of similar effective masses, as is the case for the lead salts $\mathrm{PbSe}$ and $\mathrm{PbS},{ }^{29}$ the $\mathrm{CM}$ onset occurs at higher relative photon energies, since the excess excitation energy is distributed uniformly between electrons and holes. Conversely, InSb, $\mathrm{InN}$, and $\mathrm{Si}$ all have rather large differences between $m_{e}$ and $m_{h}{ }^{29}$ (effective hole masses of $0.45-0.65 m_{0}$ have been reported for $\mathrm{InN})^{11}$ which allows for $\mathrm{CM}$ onsets closer to the ideal $2 E_{g}$. However, since the excited electron (or hole) must spend a certain amount of time in a higher energy state for $\mathrm{CM}$ to be probable, deviations from the ideal behavior may occur. Relaxation processes (see below) reduce the QY, particularly for small excess energies just above a CM threshold. ${ }^{30}$ This explains why the CM does not rise in a step like manner as in the ideal case shown in Figure 3, but rather as a straight line. Regarding (ii), the relatively small slope of the QY after the onset can be traced to processes competing with CM, specifically phonon assisted relaxation. ${ }^{1,2,31}$ Auger recombination may also contribute as a competing pathway and has indeed previously been predicted to have a negative effect upon InN based hot carrier solar cells. ${ }^{32}$ However, here the time-resolved photoconductivity data, shown in Figure 1, display no sign of decay on the probed timescale that may be associated with interband recombination. Furthermore, as described above, the observation that the photoconductivity scales linearly with excitation fluence indicates that the role of carrier-carrier recombination in the timeframe investigated is negligible. Above the CM onset, the slope of the QY vs photon energy is determined by the relative rates of the loss channels vs. impact ionization rate. While multiphonon relaxation seems to be fairly slow in $\mathrm{InN}$ (see the $\sim$ ps rise of the signal in Fig. 1), consistent with the wide phononic bandgap, ${ }^{10}$ the impact ionization rate may also be lower than in other materials. This rate is determined to an important extent by the initial (single high-energy e-h pair) and final (two e-h pairs) densities of states. ${ }^{4}$ In $\mathrm{InN}$ the valence and conduction band valleys at the $\Gamma$ point are nondegenerate, ${ }^{33}$ which may account for the low CM efficiencies observed. More detailed aspects of the energy band structure, facilitating the conservation of energy and momentum in the secondary interband transition, have also been suggested to have an impact on the CM efficiency. ${ }^{2}$

Here, the maximum power conversion efficiency from a photovoltaic device was calculated based on the materials presented in Table I using the detailed balance model, ${ }^{7}$ which takes into account the energy overlap of the QY with the solar spectrum (AM1.5G). The QY was assumed to be $100 \%$ for energies between $E_{g}$ and the CM onset and includes the experimental CM onsets and slopes for each material. For InN a conversion efficiency of $21.7 \%$ is calculated, far removed from the theoretical limit of $42.8 \%$ (calculated with ideal, step like QY and $E_{g}=0.64 \mathrm{eV}$ ). Without $\mathrm{CM}$ the conversion efficiency of an absorber with $E_{g}=0.64 \mathrm{eV}$ is $20.7 \%$, indicating that the $\mathrm{CM}$ processes observed here have the potential to increase the total conversion efficiency of an InN photovoltaic device by $1 \%$ point. This conclusion is in agreement with a recent report ${ }^{32}$

TABLE I. CM properties and solar cell efficiencies for InSb, PbSe, PbS, InN, and Si. From left to right the columns show material bandgap $E_{g}$ (eV), relative $\mathrm{CM}$ onset and slope efficiencies, solar power conversion efficiency simulated ${ }^{7}$ using the experimental CM onset and slope, and lastly the conversion efficiency increase by $\mathrm{CM}$. Data from $\mathrm{PbSe}, \mathrm{PbS}$, and $\mathrm{InN}$ were measured using THz-TDS, while data from InSb and $\mathrm{Si}$ were obtained with device current measurements.

\begin{tabular}{|c|c|c|c|c|c|}
\hline & $E_{g}(\mathrm{eV})$ & $\mathrm{CM}$ onset $/ E_{g}$ & CM slope $\times E_{g}(\%)$ & $\begin{array}{l}\text { Simulated power conversion } \\
\text { efficiency including CM }(\%)\end{array}$ & $\begin{array}{c}\text { Efficiency increase } \\
\text { caused by CM (\% point) }\end{array}$ \\
\hline $\operatorname{InSb}^{\mathrm{a}}$ & 0.17 & 2.5 & 12 & 1.50 & 1.06 \\
\hline $\mathrm{PbSe}^{\mathrm{b}}$ & 0.27 & 6.6 & 18 & 4.32 & 0.57 \\
\hline $\mathrm{PbS}^{\mathrm{b}}$ & 0.42 & 4.8 & 32 & 11.71 & 1.04 \\
\hline InN (present work) & 0.64 & $2.7 \pm 0.3$ & $13 \pm 1$ & 21.69 & 1.00 \\
\hline $\mathrm{Si}^{\mathrm{c}}$ & 1.12 & 2.9 & 25 & 33.46 & 0.05 \\
\hline
\end{tabular}

${ }^{\mathrm{a}}$ Reference 3 .

${ }^{\mathrm{b}}$ Reference 4.

${ }^{\mathrm{c}}$ Reference 1 . 
showing that owing to the competition between carrier cooling and simulated hot carrier extraction/impact ionization, the maximum efficiency of an $\mathrm{InN}$ based solar cell is indeed close to the Shockley-Queisser limit for that bandgap. From the simulated efficiencies in Table $\mathrm{I}$ it is clear that the improvements in power conversion efficiency of a PV device owing to the $\mathrm{CM}$ process are minor for the materials studied here. It has been proposed ${ }^{2}$ that tailoring the band structure of a material, for example, by alloying $\mathrm{Si}$ with $\mathrm{Ge}$, can increase rate of impact ionization, boosting the efficiency of $\mathrm{CM}$ beyond that of conventional materials. In this manner, bulk materials yielding significant PV efficiencies through CM may be attainable.

In conclusion, we employed THz-TDS to probe the carrier multiplication efficiency for excitation of $\mathrm{InN}$ between $0.95 \mathrm{eV}$ and $4.66 \mathrm{eV}$ (1300 $\mathrm{nm}$ to $266 \mathrm{~nm})$. The onset of carrier multiplication was observed at approximately $2.7 E_{g}$, and the slope of photoinduced $\mathrm{THz}$ response per absorbed photon versus photon energy was $21 \% / \mathrm{eV}$, yielding a potential $1 \%$ point increase in power conversion efficiency. While on paper InN seems like an ideal candidate for CM assisted PV applications, its true efficiency appears significantly lower than that predicted for an absorber with ideal CM properties.

This work has been financially supported by the Nederlandse Organisatie voor Wetenschappelijk Onderzoek (NWO) within the research program "Stichting voor Fundamenteel Onderzoek der Materie (FOM)." We are grateful to Professor A. Zunger for helpful discussions and Professor W. Schaff for providing the sample.

${ }^{1}$ S. Kolodinski, J. H. Werner, T. Wittchen, and H. J. Queisser, Appl. Phys. Lett. 63(17), 2405 (1993).

${ }^{2}$ J. H. Werner, S. Kolodinski, and H. J. Queisser, Phys. Rev. Lett. 72(24), 3851 (1994).

${ }^{3}$ J. Tauc, J. Phys. Chem. Solids 8, 219 (1959).

${ }^{4}$ J. J. H. Pijpers, R. Ulbricht, K. J. Tielrooij, A. Osherov, Y. Golan, C. Delerue, G. Allan, and M. Bonn, Nat. Phys. 5(11), 811 (2009).

${ }^{5}$ J. A. Mcguire, J. Joo, J. M. Pietryga, R. D. Schaller, and V. I. Klimov, Acc. Chem. Res. 41(12), 1810 (2008).

${ }^{6}$ W. Shockley and H. J. Queisser, J. Appl. Phys. 32(3), 510 (1961).

${ }^{7}$ M. C. Hanna and A. J. Nozik, J. Appl. Phys. 100(7), 074510 (2006).

${ }^{8}$ J. Wu, W. Walukiewicz, W. Shan, K. M. Yu, J. W. Ager, S. X. Li, E. E. Haller, H. Lu, and W. J. Schaff, J. Appl. Phys. 94(7), 4457 (2003).
${ }^{9}$ J. Q. Wu, J. Appl. Phys. 106(1), 011101 (2009).

${ }^{10}$ V. Y. Davydov, V. V. Emtsev, I. N. Goncharuk, A. N. Smirnov, V. D. Petrikov, V. V. Mamutin, V. A. Vekshin, S. V. Ivanov, M. B. Smirnov, and T. Inushima, Appl. Phys. Lett. 75(21), 3297 (1999).

${ }^{11}$ K. Wang, N. Miller, R. Iwamoto, T. Yamaguchi, M. A. Mayer, T. Araki, Y. Nanishi, K. M. Yu, E. E. Haller, W. Walukiewicz, and J. W. Ager, Appl. Phys. Lett. 98(4), 042104 (2011).

${ }^{12}$ R. D. Schaller, M. A. Petruska, and V. I. Klimov, Appl. Phys. Lett. 87(25), 253102 (2005).

${ }^{13}$ J. Wu, W. Walukiewicz, S. X. Li, R. Armitage, J. C. Ho, E. R. Weber, E. E. Haller, H. Lu, W. J. Schaff, A. Barcz, and R. Jakiela, Appl. Phys. Lett. 84(15), 2805 (2004).

${ }^{14}$ J. Wu, W. Walukiewicz, W. Shan, K. M. Yu, J. W. Ager, E. E. Haller, H. Lu, and W. J. Schaff, Phys. Rev. B 66(20), 201403 (2002).

${ }^{15}$ R. Ulbricht, E. Hendry, J. Shan, T. F. Heinz, and M. Bonn, Rev. Mod. Phys. 83(2), 543 (2011).

${ }^{16}$ H. Ahn, C. H. Shen, C. L. Wu, and S. Gwo, Thin Solid Films 494(1-2), 69 (2006).

${ }^{17}$ D. Fritsch, H. Schmidt, and M. Grundmann, Phys. Rev. B 69(16), 165204 (2004).

${ }^{18}$ M. C. Beard, G. M. Turner, and C. A. Schmuttenmaer, Phys. Rev. B 62(23), 15764 (2000).

${ }^{19}$ H. Ahn, Y. P. Ku, C. H. Chuang, C. L. Pan, H. W. Lin, Y. L. Hong, and S. Gwo, Appl. Phys. Lett. 92(10), 102103 (2008).

${ }^{20}$ B. Arnaudov, T. Paskova, P. P. Paskov, B. Magnusson, E. Valcheva, B. Monemar, H. Lu, W. J. Schaff, H. Amano, and I. Akasaki, Phys. Rev. B 69(11), 115216 (2004).

${ }^{21}$ S. P. Fu and Y. F. Chen, Appl. Phys. Lett. 85(9), 1523 (2004).

${ }^{22}$ M. Goiran, M. Millot, J. M. Poumirol, I. Gherasoiu, W. Walukiewicz, and J. Leotin, Appl. Phys. Lett. 96(5), 052117 (2010).

${ }^{23}$ M. Millot, N. Ubrig, J. M. Poumirol, I. Gherasoiu, W. Walukiewicz, S. George, O. Portugall, J. Leotin, M. Goiran, and J. M. Broto, Phys. Rev. B 83(12), 125204 (2011).

${ }^{24}$ S. K. Pugh, D. J. Dugdale, S. Brand, and R. A. Abram, Semicond. Sci. Tech. 14(1), 23 (1999).

${ }^{25}$ G. Pettinari, A. Polimeni, M. Capizzi, J. H. Blokland, P. C. M. Christianen, J. C. Maan, V. Lebedev, V. Cimalla, and O. Ambacher, Phys. Rev. B 79(16), 165207 (2009).

${ }^{26}$ A. Kasic, M. Schubert, Y. Saito, Y. Nanishi, and G. Wagner, Phys. Rev. B 65(11), 115206 (2002).

${ }^{27}$ T. Inushima, T. Shiraishi, and V. Y. Davydov, Solid State Commun. 110(9), 491 (1999).

${ }^{28}$ E. Hendry, M. Koeberg, J. Pijpers, and M. Bonn, Phys. Rev. B 75(23), 233202 (2007).

${ }^{29}$ O. Madelung, Semiconductors: Data Handbook, 3rd ed. (Springer, Germany, 2004).

${ }^{30}$ A. Franceschetti, J. M. An, and A. Zunger, Nano Lett. 6(10), 2191 (2006).

${ }^{31}$ J. W. Luo, A. Franceschetti, and A. Zunger, Nano Lett. 8(10), 3174 (2008).

${ }^{32}$ P. Aliberti, Y. Feng, S. K. Shrestha, M. A. Green, G. Conibeer, L. W. Tu, P. H. Tseng, and R. Clady, Appl. Phys. Lett. 99(22), 223507 (2011).

${ }^{33}$ P. Aliberti, Y. Feng, Y. Takeda, S. K. Shrestha, M. A. Green, and G. Conibeer, J. Appl. Phys. 108(9), 094507 (2010). 